\title{
Pfhrp2 and pfhrp3 polymorphisms in Plasmodium falciparum isolates from Dakar, Senegal: impact on rapid malaria diagnostic tests
}

Nathalie Wurtz ${ }^{1,2}$, Bécaye Fall ${ }^{3}$, Kim Bui ${ }^{1,2}$, Aurélie Pascual ${ }^{1,2,4}$, Mansour Fall ${ }^{5}$, Cheikhou Camara ${ }^{6}$, Bakary Diatta ${ }^{5}$, Khadidiatou Ba Fall ${ }^{7}$, Pape Saliou Mbaye ${ }^{8}$, Yaya Diémé ${ }^{3}$, Raymond Bercion ${ }^{3}$, Boubacar Wade ${ }^{9}$, Sébastien Briolant ${ }^{1,2}$ and Bruno Pradines ${ }^{1,2,3,4^{*}}$

\begin{abstract}
Background: An accurate diagnosis is essential for the rapid and appropriate treatment of malaria. The accuracy of the histidine-rich protein 2 (PfHRP2)-based rapid diagnostic test (RDT) Palutop $+4^{\circledR}$ was assessed here. One possible factor contributing to the failure to detect malaria by this test is the diversity of the parasite PfHRP2 antigens.

Methods: PfHRP2 detection with the Palutop $+4{ }^{\circledR}$ RDT was carried out. The pfhrp2 and pfhrp3 genes were amplified and sequenced from 136 isolates of Plasmodium falciparum that were collected in Dakar, Senegal from 2009 to 2011. The DNA sequences were determined and statistical analyses of the variation observed between these two genes were conducted. The potential impact of PfHRP2 and PfHRP3 sequence variation on malaria diagnosis was examined.

Results: Seven P. falciparum isolates (5.9\% of the total isolates, regardless of the parasitaemia; $10.7 \%$ of the isolates with parasitaemia $\leq 0.005 \%$ or $\leq 250$ parasites/ $\mu$ l) were undetected by the PfHRP2 Palutop $+4^{\circledR}$ RDT. Low parasite density is not sufficient to explain the PfHRP2 detection failure. Three of these seven samples showed pfhrp2 deletion (2.4\%). The pfhrp3 gene was deleted in 12.8\%. Of the 122 PfHRP2 sequences, 120 unique sequences were identified. Of the 109 PfHRP3 sequences, 64 unique sequences were identified. Using the Baker's regression model, at least $7.4 \%$ of the P. falciparum isolates in Dakar were likely to be undetected by PfHRP2 at a parasite density of $\leq 250$ parasites/ $\mu \mathrm{l}$ (slightly lower than the evaluated prevalence of 10.7\%). This predictive prevalence increased significantly between 2009 and $2011(P=0.0046)$.

Conclusion: In the present work, $10.7 \%$ of the isolates with a parasitaemia $\leq 0.005 \%$ ( $\leq 250$ parasites $/ \mu$ l) were undetected by the PfHRP2 Palutop $+4{ }^{\circledR}$ RDT (7.4\% by the predictive Baker'model). In addition, all of the parasites with pfhrp 2 deletion (2.4\% of the total samples) and $2.1 \%$ of the parasites with parasitaemia $>0.005 \%$ and presence of pfhrp2 were not detected by PfHRP2 RDT. PfHRP2 is highly polymorphic in Senegal. Efforts should be made to more accurately determine the prevalence of non-sensitive parasites to pfHRP2.
\end{abstract}

Keywords: Malaria, Plasmodium falciparum, Diagnosis, Rapid diagnostic test, Plasmodium falciparum histidine-rich protein 2, Anti-malarial

\footnotetext{
* Correspondence: bruno.pradines@free.fr

'Unité de Parasitologie, Département d'Infectiologie de Terrain, Institut de Recherche Biomédicale des Armées, Marseille, France

${ }^{2}$ Aix Marseille Université, Unité de Recherche sur les Maladies Infectieuses et Tropicales Emergentes, UM 63, CNRS 7278, IRD 198, Inserm 1095, Marseille,

France

Full list of author information is available at the end of the article
} 


\section{Background}

The early and accurate diagnosis of malaria is important for the effective management and treatment of this disease to reduce morbidity and mortality. Malaria diagnosis relies on the microscopic examination of blood smears, which remains the standard method. The World Health Organization (WHO) recently recommended the adoption of universal testing to confirm the presence of malaria parasites prior to the use of artemisinin-based combination therapy (ACT). In cases where microscopic examination cannot be performed, rapid diagnostic tests (RDTs) are the best alternative for diagnosis. RDTs can be useful because they provide quick and accurate diagnoses, thereby leading to timely and accurate treatment and reducing the severity and economic burden of the disease. Today, many RDTs are commercially available, and they utilize immunochromatographic methods to detect parasite-specific antigens in lysed blood cells [1]. Most products detect Plasmodium falciparum-specific proteins and target either the P. falciparum histidine-rich protein 2 (PfHRP2) or the $P$. falciparum lactate dehydrogenase (PfLDH). Certain RDTs can detect both $P$. falciparum-specific and pan-specific antigens (aldolase (pALD) or pan-malaria (pLDH)) [1]. Many factors related either to the parasite or to the test may affect the performance of malaria RDTs. Although pALD and pLDH appear to be highly conserved $[2,3]$, it has been reported that $p f h r p 2$ sequence variations, particularly with regard to certain amino acid repeats, can affect the sensitivity of HRP2-based RDTs [3-7]. These polymorphisms have been detected in the Asia-Pacific region [4,5], India [6], Madagascar [3] and in a clinical case in Uganda [7]. In addition, misdiagnosis may also arise from gene deletions that prevent the expression of proteins by the parasite. More recently, the deletions of pfhrp 2 and pfhrp3 were reported as the causes of falsenegative diagnoses in populations from Peru [8,9], Mali [10], India [11] and in a clinical case from Brazil [12].

The aims of this study were to investigate the genetic diversity of the $p f h r p 2$ and $p f h r p 3$ genes in P. falciparum clinical isolates from Dakar, Senegal, and its impact on predictive HRP2-based RDT diagnostic sensitivity using the Baker's regression model [4]. Then, predictive results were compared to results obtained with use of the HRP2-based RDT Palutop $+4^{\circledR}$ (All Diag, Strasbourg, France), microscopic examination and real-time polymerase chain reaction.

\section{Methods}

\section{Plasmodium falciparum isolates}

A total of 136 blood samples were selected for analysis from the Hôpital Principal de Dakar. The samples, stored at $-20^{\circ} \mathrm{C}$, were from patients who came to the hospital for a malaria consultation and were recruited for the evaluation of $P$. falciparum ex vivo susceptibility to anti-malarial drugs [13-15]. The samples represented three malaria seasons: 14 October 2009 to 19 January 2010, 20 July 2010 to 18 February 2011 and 3 October 2011 to 13 January 2012. Informed verbal consent was obtained from patients or their parents prior to blood collection. All the tests were performed on the same blood samples used for the malaria diagnosis. The study was reviewed and approved by the ethics committee of the Hôpital Principal de Dakar.

\section{Diagnosis of malaria}

The diagnosis of malaria was assessed by microscopic examination, use of HRP2-based RDT and real-time polymerase chain reaction. At recruitment, thin blood smears were stained using a RAL ${ }^{\circledR}$ kit (Réactifs RAL, Paris, France) and were examined to determine the parasite density and diagnosis.

Immunochromatographic rapid tests (RDT Palutop $+4^{\circledR}$ ) were used to perform the diagnosis for four species of Plasmodium. Palutop $+4^{\circledR}$ is a rapid test that uses whole blood for the detection of the P. falciparum-specific histidine rich protein-2 (PfHRP2), the Plasmodium vivax-specific lactate dehydrogenase (LDH) and the pan-malariaspecific $\mathrm{pLDH}$ for $P$. falciparum, $P$. vivax, Plasmodium ovale or Plasmodium malariae (Figure 1).

To confirm the diagnosis obtained by the RDTs and the blood smear examination, $P$. falciparum and $P$. vivax detection was performed by real-time PCR using a LightCycler ${ }^{\circledR}$ (Roche, Meylan, France). The following oligonucleotide primers and probes, designed with Primer Express software v2.0 (Applied Biosystems), were used: forward 5'-TTTATGTATTGGTATAATTCGG3', reverse-5' - GGCAAATAACTTTATCATAGAATTG AC-3' and probe-5'-FAM-TACACTACCAACACATG GGGCTACAAGAGGT-BBQ-3' for the $P$. falciparum aquaglyceroporin gene (AJ413249); forward-5'-GTGGC CGCCTTTTTGCT-3', reverse-5'-CCTCCCTGAAACAA GTCATCG-3' and probe-5'-HEX-CATCTACGTGGACA ACGGGCTCAACA-BHQ1-3' for the P. vivax enoyl-acyl carrier protein reductase gene (AY423076) (Eurogentec, Angers, France). Each parasite species was detected separately. The individual real-time PCR amplifications were carried out using the previously described conditions [16].

\section{Nucleic acid extraction}

Total genomic DNA of each strain was isolated using the QIAamp ${ }^{\circledR}$ DNA Mini kit according to the manufacturer's recommendations (Qiagen, Germany).

\section{pfhrp2 sequencing}

A 905-nucleotide fragment of exon 2 of the $P$. falciparum histidine-rich protein 2 (pfhrp2) gene (M13986) was amplified by PCR using the pfhrp2-sense 5'- TGTGTAGCAAAAATGCAAAAGG -3' and the pfhrp2-antisense 


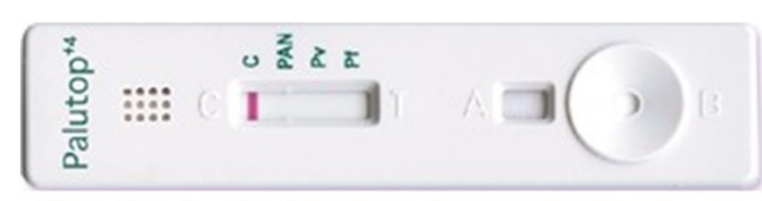

Negative for the four species Only one band appears in C (control)

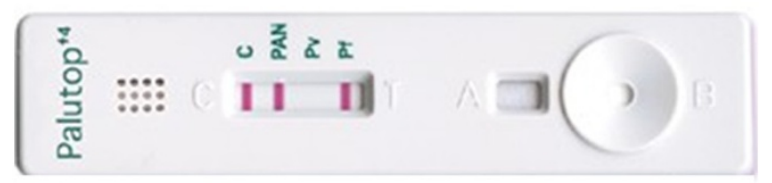

Positive for Plasmodium falciparum In addition to the control band, $\mathbf{a}$ band appears in Pf and Pan, respectively

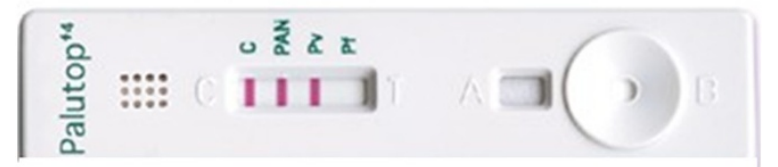

Positive for Plasmodium vivax In addition to the control band, $\mathbf{a}$ band appears in $\mathrm{PV}$ and $\mathrm{Pan}$, respectively

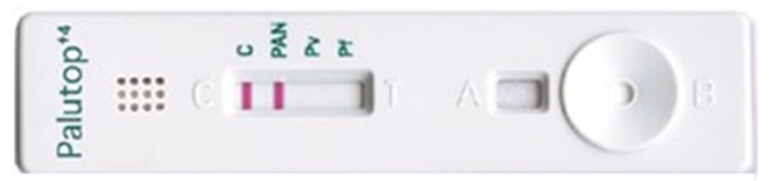

Positive for other Plasmodium In addition to the control band, $\mathbf{a}$ band appears in Pan

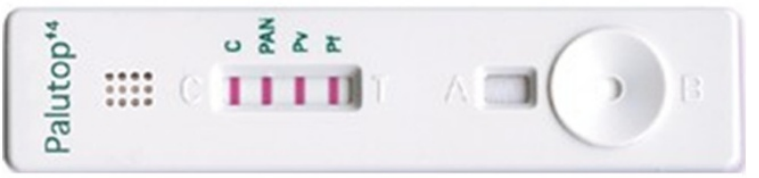

Positive for multiple infection In addition to the control band, $\mathbf{a}$ band appears in $P f, P v$ and $P$ an, respectively

Figure 1 Malaria diagnosis by either the Palutop $+4{ }^{\circledR}$ rapid diagnostic test, which recognizes the Plasmodium falciparumspecific histidine rich protein-2 (PfHRP2), the Plasmodium vivax-specific LDH test for $P$. vivax detection and the panmalaria-specific pLDH test for the detection of $P$. falciparum, $P$. vivax, Plasmodium ovale or Plasmodium malariae.

5' - TTAATGGCGTAGGCAATGTG -3' primers [7]. The reaction mixture for the PCR amplification included 5 $\mu \mathrm{l}$ of genomic DNA, $2.5 \mu \mathrm{l}$ of $10 \mathrm{X}$ reaction buffer (Eurogentec, Angers, France), $0.5 \mu \mathrm{M}$ of each primer, $200 \mu \mathrm{M}$ of a deoxynucleoside triphosphate mixture (dGTP, dATP, dTTP and dCTP) (Euromedex, Souffelweyersheim, France), $1.5 \mathrm{mM} \mathrm{MgCl} 2$ and 1 unit of RedGoldStar ${ }^{\circledR}$ DNA polymerase (Eurogentec, Angers, France) to a final volume of $25 \mu \mathrm{l}$. The thermal cycler (T3 Biometra, Archamps, France) was programmed as follows: $94^{\circ} \mathrm{C}$ for $5 \mathrm{~min} ; 45$ cycles of $94^{\circ} \mathrm{C}$ for $30 \mathrm{sec}, 62^{\circ} \mathrm{C}$ for $40 \mathrm{sec}$ and $72^{\circ} \mathrm{C}$ for 90 sec; 10 min extension step at $72^{\circ} \mathrm{C}$. The PCR products were loaded on a $1 \%$ agarose gel containing $0.5 \mu \mathrm{g} / \mathrm{mL}$ ethidium bromide. The amplicons were purified using the High Pure 96 UF Cleanup Kit according to the manufacturer's instructions (Roche). The purified fragments were sequenced using the BigDye Terminator v3.1 Cycle Sequencing Kit (Applied Biosystems) using the primers described above. The sequencing reaction products were purified using the BigDye XTerminator ${ }^{\circledR}$ Purification Kit (Applied Biosystems) in accordance with the manufacturer's instructions. The purified products were sequenced using an ABI Prism 3100 analyzer (Applied Biosystems). The sequences were analysed and translated using the Vector NTI advance (TM) software (version 11, Invitrogen, Cergy Pontoise, France). The amino acid repeats were identified by numerical code (1-18) as described by Baker et al. [5].

\section{pfhrp3 sequencing}

A 727-nucleotide fragment of exon 2 of the $P$. falciparum histidine-rich protein 3 (pfhrp3) gene (U69552) was amplified by PCR using the pfhrp3-sense 5'- TG TTTAGCAAAAATGCAAAAGGACT -3' and pfhrp3antisense 5'- TGTAAGTGATGCGTAGTGGCA -3' primers (Eurogentec, Angers, France), which were designed with the NCBI/Primer-BLAST online tool. The reaction mixture for the PCR amplification included $2 \mu \mathrm{l}$ of genomic DNA, $2.5 \mu \mathrm{l}$ of $10 \mathrm{X}$ reaction buffer (Eurogentec, Angers, France), $0.5 \mu \mathrm{M}$ of each primer, $200 \mu \mathrm{M}$ of a deoxynucleoside triphosphate mixture (dGTP, dATP, dTTP and dCTP) (Euromedex, Souffelweyersheim, France), 1.5 $\mathrm{mM} \mathrm{MgCl} 2$ and 1 unit of RedGoldStar ${ }^{\circledR}$ DNA polymerase (Eurogentec, Angers, France) in a final volume of 25 $\mu$ l. The thermal cycler (T3 Biometra, Archamps, France) was programmed as follows: $94^{\circ} \mathrm{C}$ for $5 \mathrm{~min}$; 40 cycles of $94^{\circ} \mathrm{C}$ for $30 \mathrm{sec}, 54^{\circ} \mathrm{C}$ for $40 \mathrm{sec}$ and $72^{\circ} \mathrm{C}$ for $50 \mathrm{sec}$; 10 min extension step at $72^{\circ} \mathrm{C}$. The PCR products were loaded on a $1 \%$ agarose gel containing $0.5 \mu \mathrm{g} / \mathrm{mL}$ ethidium bromide. The amplicons were purified using the High Pure 96 UF Cleanup Kit according to the manufacturer's instructions (Roche). The purified fragments were sequenced using the BigDye Terminator v3.1 Cycle Sequencing Kit (Applied Biosystems) using the primers described above. The sequencing reaction products were purified as described in the previous section.

\section{Assessment of pfhrp2 and pfhrp3 gene deletions}

To confirm gene deletion and not negative PCR reactions due to poor quality of DNA, independent PCR experiments were assessed on samples with no amplicon detected by amplification of entire exon 2 of $p f h r p 2$ or $p f h r p 3$, described in the previous sections. Further amplifications were performed across exon 1 and 2 of both pfhrp 2 and $p f h r p 3$ using primers previously described [8]. 


\section{Data analysis and statistical tests}

The number of repeats were determined for the $\mathrm{AHH}$ AHHVAD pattern (type 1), AHHAHHAAD (type 2), AH HAHHAAY (type 3), AHH (type 4), AHHAHHASD (type 5), AHHATD (type 6), AHHAAD (type 7), AHHAAY type 8), AAY (type 9), AHHAAAHHATD (type 10), AHN (type 11), AHHAAAHHEAATH (type 12), AHHASD (type 13) and AHHAHHATD (type 14) for PfHRP2.

PfHRP2 sequences were classified into four groups as a function of the number of type $2 \times$ type 7 repeats: group A PfHRP2 sequences (very sensitive) contained more than 100 type $2 \times$ type 7 repeats, group B (sensitive) contained between 50-100 type $2 \times$ type 7 repeats, group $C$ (non-sensitive) contained $<43$ repeats and "borderline" group I contained between 44 and 49 repeats.

The difference in the percentage of isolates belonging to group $\mathrm{C}$ for each malaria season and of detected or undetected parasites was analysed by the Chi-square test. The parasitaemia was classified into two groups: parasitaemia $\leq 0.005 \%$ and $>0.005 \%$. The association between the absence of a diagnosis by PfHRP2 and the particular group of HRP2 sensitivity, parasitaemia or type of amino acid repeat was analysed using the Wilcoxon rank sum test and the Kruskal-Wallis rank sum test.

\section{Results}

\section{Plasmodium falciparum diagnoses}

The parasitaemia ranged from 0.0000 to $15.8 \%$ (median $=$ $0.1 \%$, Q1 $=0.02 \%$; Q3 = 0.5\%). 22.8\% of the samples showed a parasitaemia $\leq 0.005 \%(\leq 250$ parasites $/ \mu \mathrm{L})$.

\section{Plasmodium falciparum false-negative diagnoses by the PfHRP2-based Palutop $+4^{\circledR}$}

Of 118 isolates, regardless of parasite density, seven samples were not detected by the PfHRP2-based Palutop $+4{ }^{\circledR}$. Three showed a parasitaemia $\leq 0.005 \%(\leq 250$ parasites $/ \mu \mathrm{l})$. There was no significant difference in term of sample numbers ( $\mathrm{p}=0.246$, Chi-square test) or in term of parasitaemia between detected (mean $=0.45 \%$; standard deviation $=1.16 \%)$ and undetected samples $($ mean $=$ $0.22 \%$; standard deviation $=0.26 \%)(\mathrm{p}=0.595$, Wilcoxon rank sum test). However, one of these samples was detected by the pan-malaria-specific $\mathrm{pLDH}$ and the $P$. vivax-specific LDH (Table 1). All of the seven samples were detected as $P$. falciparum by real-time polymerase chain reaction.

Of the isolates detected as P. falciparum by PfHRP2based Palutop $+4^{\circledR}, 7.1 \%$ were not detected by the panmalaria-specific pLDH. The parasitaemia ranged from $0.0000 \%$ to $0.5 \%$ (mean $=0.18 \%$; standard deviation $=$ $0.24 \%$ ) for the undetected group and $0.0000 \%$ to $15.8 \%$ ( mean $=0.48 \%$; standard deviation $=1.20 \%$ ) for the group detected by the pan-malaria-specific pLDH. There was no significantly difference in term of parasitaemia between the two groups ( $\mathrm{p}=0.144$, Wilcoxon rank sum test).

\section{Plasmodium vivax false-positive diagnoses the PfHRP2-based Palutop $+4^{\circledR}$}

Five of 118 samples were detected as $P$. vivax by the RDT Palutop $+4^{\circledR}$ (Table 2). These five samples were suspected as $P$. falciparum by microscopy and were confirmed as $P$. falciparum by real-time polymerase chain reaction. None of these samples was confirmed as $P$. vivax. These five samples showed a parasitaemia $\geq 0.005 \%$.

\section{Genetic diversity of Plasmodium falciparum HRP2 and HRP3} For PfHRP2, 122 PCR fragments were successfully amplified. No amplicon was detected for three samples (186/ $1,56 / 3$ and $150 / 3$ ) by the two methods. The pfhrp 2 gene was deleted in these three samples (2.4\% of the samples). Thirteen of the 14 previously identified amino acid repeats were detected [4] (Table 3). Of the 122 PfHRP2 sequences, 120 unique sequences were identified. Only three PfHRP2 sequences did not begin with the type 1 repeat (AHHAHHVAD). The type 2 repeat (AHHAHHAAD)

Table 1 Plasmodium falciparum false-negative diagnoses by the HRP2-based test

\begin{tabular}{|c|c|c|c|c|c|c|c|c|c|}
\hline \multirow[b]{2}{*}{ Identification } & \multicolumn{3}{|c|}{ Palutop $+4^{\circledR}$} & \multicolumn{2}{|c|}{ Microscopy } & \multicolumn{2}{|c|}{ RT-PCR } & \multirow[t]{2}{*}{$\mathrm{HRP}^{\mathrm{a}}{ }^{\mathrm{a}}$} & \multirow[t]{2}{*}{ HRP3 $^{b}$} \\
\hline & Pan & Pf & PV & Diagnostic & Parasitaemia & Pf & $P v$ & & \\
\hline $186 / 1$ & + & - & + & $P f$ & $0.5 \%$ & + & - & - & - \\
\hline $212 / 1$ & - & - & - & $\mathrm{Pf}$ & $0.02 \%$ & + & - & $+(B)$ & - \\
\hline $55 / 2$ & - & - & - & $\mathrm{Pf}$ & $0.5 \%$ & + & - & $+(B)$ & - \\
\hline $56 / 3$ & - & - & - & $P f$ & $0.5 \%$ & + & - & - & + \\
\hline $150 / 3$ & - & - & - & $\mathrm{Pf}$ & $0.001 \%$ & + & - & - & - \\
\hline $157 / 3$ & - & - & - & $-^{c}$ & & + & - & $+(B)$ & - \\
\hline $183 / 3$ & - & - & - & Pf & $0.004 \%$ & + & - & $+(C)$ & - \\
\hline
\end{tabular}

$\mathrm{Pf}=$ Plasmodium falciparum; $\mathrm{Pv}=$ Plasmodium vivax $; \mathrm{RT}-\mathrm{PCR}=$ real-time polymerase chain reaction

a - If deletion of pfhrp2 and + if presence of pfhrp2.

(B) Group B, sensitive to PfHRP2, detection, number of type 2 repeats $\times$ type 7 repeats between 50 to 100 repeats.

(C) Group C, non sensitive PfHRP2 detection, number of type 2 repeats $\times$ type 7 repeats $<43$ repeats.

b - If deletion of pfhrp3 and + if presence of pfhrp3.

c Thin and thick blood smears were negative. 
Table 2 Plasmodium vivax false-positive diagnoses

\begin{tabular}{lcccccccc}
\hline & \multicolumn{3}{c}{ Palutop $^{4}$ ( $^{\circledR}$} & \multicolumn{3}{c}{ Microscopy } & \multicolumn{2}{c}{ RT-PCR } \\
\hline Identification & Pan & Pf & PV & Diagnostic & Parasitaemia & Pf & PV \\
$146 / 1$ & - & + & + & Pf & $0.8 \%$ & + & - \\
$186 / 1$ & + & - & + & Pf & $0.5 \%$ & + & - \\
$81 / 3$ & + & + & + & Pf & $1.4 \%$ & + & - \\
$113 / 3$ & + & + & + & Pf & $2.0 \%$ & + & - \\
$146 / 3$ & + & + & + & Pf & $0.06 \%$ & + & - \\
\hline
\end{tabular}

$\mathrm{Pf}=$ Plasmodium falciparum; $\mathrm{Pv}=$ Plasmodium vivax $; \mathrm{RT}-\mathrm{PCR}=$ real-time polymerase chain reaction.

was observed in $100 \%$ of the sequenced isolates, the type 3 (AHHAHHAAY) in $89 \%$, the type 5 (AHHAHHASD) in 75\%, the type 6 (AHHATD) in 98\%, the type 7 (AHHAAD) in 99\%, the type 8 (AHHAAY) in $84 \%$ and the type 10 (AHHAAAHHATD) in 75\%. Several of the repeats occurred in only a few isolates: the type $4(\mathrm{AHH})$ in 20 isolates (16\%), the type 9 (AAY) in two isolates, the type 12 (AHHAAAHHEAATH) in only one isolate, the type 13 (AHHASD) in nine isolates and the type 14 (AHHAHHATD) in five isolates. The type 11 repeat (AHN) was not detected in P. falciparum parasites from Dakar.

The PfHRP2 sequences were classified as described in the Methods section. The results of the classification of the PfHRP2 sequences into type $2 \times$ type 7 repeat groups are shown in Table 4.

Table 3 The distribution of amino acid repeats (minimum to maximum number) in PfHRP2 and PfHRP3 from Plasmodium falciparum isolates from Dakar, Senegal

\begin{tabular}{clcc}
\hline Code & Amino-acid repeats & PfHRP2 & PfHRP3 \\
\hline 1 & AHHAHHVAD & $0-8$ & $0-4$ \\
2 & AHHAHHAAD & $4-17$ & 0 \\
3 & AHHAHHAAY & $0-3$ & 0 \\
4 & AHH & $0-3$ & 1 \\
5 & AHHAHHASD & $0-2$ & 0 \\
6 & AHHATD & $0-7$ & 0 \\
7 & AHHAAD & $0-14$ & $1-2$ \\
8 & AHHAAY & $0-2$ & 0 \\
9 & AAY & $0-1$ & 0 \\
10 & AHHAAAHHATD & $0-3$ & 0 \\
11 & AHN & 0 & 0 \\
12 & AHHAAAHHEAATH & $0-1$ & 0 \\
13 & AHHASD & $0-2$ & 0 \\
14 & AHHAHHATD & $0-1$ & 0 \\
15 & AHHAHHAAN & 0 & $0-1$ \\
16 & AHHAAN & 0 & $3-19$ \\
17 & AHHDG & 0 & $3-9$ \\
18 & AHHDD & 0 & $0-6$ \\
\hline
\end{tabular}

Table 4 The distribution of PfHRP2 sequences (number, no; percentage, \%) into four groups based on the number of type $2 \times$ type 7 repeats: group A (very sensitive) PfHRP2 sequences contained more than 100 type $2 \times$ type 7 repeats, group B (sensitive) contained between 50 to 100 type $2 \times$ type 7 repeats, group $C$ (non-sensitive) contained $<43$ repeats and the "borderline" group I contained between 44 and 49 repeats

\begin{tabular}{ccccccccc}
\hline & \multicolumn{2}{c}{ Group A } & \multicolumn{2}{c}{ Group B } & \multicolumn{2}{c}{ Group I } & \multicolumn{2}{c}{ Group C } \\
\hline Year & no & $\%$ & no & $\%$ & no & $\%$ & no & $\%$ \\
$2009-2010$ & 9 & 23.1 & 30 & 76.9 & 0 & 0 & 0 & 0 \\
$2010-2011$ & 7 & 17.5 & 29 & 72.5 & 2 & 5 & 2 & 5 \\
$2011-2012$ & 5 & 11.6 & 28 & 65.1 & 3 & 7.0 & 7 & 16.3 \\
$\mathbf{2 0 0 9 - 2 0 1 2}$ & $\mathbf{2 1}$ & $\mathbf{1 7 . 2}$ & $\mathbf{8 7}$ & $\mathbf{7 1 . 3}$ & $\mathbf{5}$ & $\mathbf{4 . 1}$ & $\mathbf{9}$ & $\mathbf{7 . 4}$ \\
\hline
\end{tabular}

The number of isolates belonging to group $\mathrm{C}$ (nonsensitive to the HRP2 test) increased significantly between 2009 and 2011 ( $P=0.0046$, Chi-square test).

For PfHRP3, 109 PCR fragments were successfully amplified. No amplicon was detected for 16 samples by the two methods. The pfhrp3 gene was deleted in $12.8 \%$ of the samples. Seven of the eight previously identified amino acid repeats were detected [4] (Table 3). Of the 109 PfHRP3 sequences, 64 unique sequences were identified. The type 1 repeat (AHHAHHAAD) was observed in $99 \%$ of the sequenced isolates, the type $4(\mathrm{AHH})$ in 100\%, the type 7 (AHHAAD) in 100\%, the type 15 (AHHAHHAAN) in 99\%, the type 16 (AHHAAN) in $100 \%$, the type 17 (AHHDG) in 100\% and the type 18 (AHHDD) in 99\%. The type 2 repeat (AHHAHHAAD) was not detected in P. falciparum parasites from Dakar.

\section{Comparison between the HRP2-based RDT results and} genetic polymorphisms or expected frequency determined by the Baker's regression model The three samples (186/1, 56/3 and 150/3) with deletion of pfhrp2 (2.4\%) were not detected by the PfHRP2-based Palutop $+4{ }^{\circledR}$. One of these three samples $(186 / 1)$ was detected by the pan-malaria-specific pLDH. Of the 16 samples with deletion of pfhrp3 (12.8\%), six were not detected by the PfHRP2-based Palutop $+4{ }^{\circledR}$.

Of the four samples not detected by the PfHRP2-based Palutop $+44^{\circledR}$ but with PfHRP2, only one (183/3) was classified in group $\mathrm{C}$ (non-sensitive) according to the Baker's regression model. In addition, of the nine samples expected to be not detected by PfHRP2-based RDT, the sample $183 / 3$ was the only one which was not detected by the PfHRP2-based Palutop $+4^{\circledR}$. There was no significant difference in parasitaemia between the four predict groups ( $\mathrm{p}=0.676$, Kruskal-Wallis rank sum test). However, there was a significant association between the absence of a diagnosis by PfHRP2 RDT and the number of type 2 repeats (AHHAHHAAD) $(P=0.0463)$. 
However, there was a significant association between the absence of a diagnosis by PfHRP2 RDT and the number of type 2 repeats (AHHAHHAAD) $(P=0.0463)$. There was no significant association between the absence of a diagnosis by PfHRP2-based Palutop $+4^{\circledR}$ and the genetic diversity of other PfHRP2 repeats: type 1 (AHHAHH VAD) $(P=0.4634$, Fisher's Exact test), type 3 (AHHAH HAAY $)(P=0.7852)$, type $4(\mathrm{AHH})(P=1)$, type 5 (AHHAHHASD) $(P=0.7589)$, type 6 (AHHATD) $(P=$ $0.1959)$, type 7 (AHHAAD) $(P=0.1872)$, type 8 (AH HAAY $)(P=1)$, type 9 (AAY) $(P=1)$, type $10(\mathrm{AH}$ HAAAHHATD) $(P=0.8402)$, type 12 (AHHAAAHHE AATH $)(P=1)$, type 13 (AHHASD) $(P=1)$ and type 14 (AHHAHHATD) $(P=1)$.

Among the 109 sequences of HRP3, only one isolate was not detected by PfHRP2-based Palutop $+4^{\circledR}$. There was no association between the absence of a diagnosis by PfHRP2-based Palutop $+4^{\circledR}$ and the genetic diversity of PfHRP3.

\section{Discussion}

The WHO recently recommended adopting universal testing to confirm the presence of malaria parasites prior to the use of ACT, and in the cases where microscopic examination cannot be performed, the RDT would be the best alternative for confirmation. In 2006, the Senegalese National Malaria Control Programme recommended $\mathrm{ACT}$ as the first-line treatment for uncomplicated malaria and, in 2007, mandated testing for all suspected cases of malaria with P. falciparum using the HRP2-based RDT. PfHRP2-based RDTs are now commonly used with high adherence in Senegal [17-19]. These RDTs can provide a quick and accurate diagnosis, thereby leading to timely and appropriate malaria treatment and a reduction in the severity and economic burden of the disease.

In the present work, $5.9 \%$ of the total $(n=7)$ P. falciparum isolates and $10.7 \%$ of the isolates with parasitaemia $\leq 0.005 \%$ ( $\leq 250$ parasites $/ \mu \mathrm{l})$ were undetected by the PfHRP2 Palutop $+4^{\circledR}$ RDT. Moreover, of the isolates detected as $P$. falciparum by PfHRP2, 7.1\% were not detected by the pan-malaria-specific pLDH. Five $P$. falciparum isolates $(4.2 \%)$ were misdiagnosed as $P$. vivax parasites by the $P$. vivax LDH.

Three possible factors can affect the sensitivity of the PfHRP2-based RDT: parasite density, pfhrp2 deletion and pfhrp 2 polymorphisms. The parasite density can not explain the failure of the detection by the PfHRP2 Palutop $+4^{\circledR}$ RDT. There was no significant difference in term of sample numbers ( $p=0.246$, Chi-square test) or in term of parasitaemia between detected (mean $=0.45 \%$; standard deviation $=1.16 \%$ ) and undetected samples by HRP2 $($ mean $=0.22 \%$; standard deviation $=0.26 \%)(\mathrm{p}=0.595$, Wilcoxon rank sum test). In addition, the non-detection by the pan-malaria-specific pLDH was not associated with parasite density. There was no significantly difference in term of parasitaemia between the two groups $(\mathrm{p}=0.144$, Wilcoxon rank sum test). For the five P. falciparum isolates $(4.2 \%)$ misdiagnosed as $P$. vivax parasites by the $P$. vivax $\mathrm{LDH}$, all of these showed parasitaemia $\geq 0.005 \%$ ( $\geq 250$ parasites $/ \mu \mathrm{l})$.

Another possible factor affecting the sensitivity of the PfHRP2-based RDT is the failure of the parasite to express the antigen, either due to genetic deletions, frame shift mutations or alterations in protein expression. The deletions of pfhrp 2 and pfhrp 3 were reported as the cause of false-negative diagnoses in populations from Peru [8,9], Mali [10], India [11] and in a clinical case from Brazil [12]. In addition, the levels of pfhrp2 transcription and PfHRP2 protein expression varied between $P$. falciparum strains, and this may impact the detection sensitivity of the PfHRP2-based RDT [20]. The three samples with deletion of pfhrp2 (2.4\%) were not detected by the PfHRP2-based Palutop $+4^{\circledR}$. The deletion of pfhrp2 is one of the factors of false-negative diagnoses using PfHRP2-based RDT. Of the 16 samples with deletion of pfhrp3 (12.8\%), six were not detected by the PfHRP2-based Palutop $+4^{\circledR}$. The frequency of $p f h r p 2$ deletion observed in Senegal is comparable to those estimated in Africa, like in Mali (2\%) [10] or in India (4.2\%) [11] and lower than those estimated in South America like in Peru (25.7 and 41\%) [8,9]. As previously described in Peru [8], the proportion of parasites lacking pfhrp3 is higher than those lacking pfhrp2 in samples collected in Dakar. This suggests that parasites lacking pfhrp3 may have been present in Senegal longer than those lacking pfhrp2.

Polymorphisms in the pfhrp 2 gene that can affect the sensitivity of PfHRP2-based RDTs have been detected in the Asia-Pacific region [4,5], India [3], Madagascar [3] and in a clinical case in Uganda [7]. Prior to this report, no data were yet available on the sequence variation of HRP2 from P. falciparum parasites from Senegal. Consistent with previous reports [5-7], the PfHRP2 was highly diverse in parasite isolates from Dakar. Of the 122 PfHRP2 sequences, 120 unique sequences were identified. In previous works, all $p f h r p 2$ sequences have begun with a type 1 repeat (AHHAHHVAD) [5-7]. However, three of the Senegalese isolate sequences in this study did not begin with a type 1 repeat. The type 11 repeat (AHN) was not detected in P. falciparum parasites from Dakar. This is consistent with isolates from Africa [4], except for one isolate from Cameroon [5] and one from northern Madagascar [3]. However, the Senegalese PfHRP2 sequences had certain characteristics: only one isolate possessed a type 12 repeat (AHHAAAHHEAATH), while the PfHRP2 from all the previously described isolates from Africa, the Pacific, South America or Asia contained one type 12 repeat $[3,5]$. The type 9 repeat has not been described in Africa 
[3-5]. Interestingly, two isolates from Dakar showed a type 9 repeat. Types 13 and 14 have rarely been observed in Africa [3-5], yet these two types of repeats were found in nine $(7.4 \%)$ and five $(4.1 \%)$ isolates from Dakar, respectively.

Finally, using the Baker's regression model, it was shown that at least $7.4 \%$ of P. falciparum isolates in Dakar (group C) were likely to be undetected by PfHRP2-based RDT at a parasite density of $\leq 250$ parasites/ $\mu$ l. In Madagascar, $9 \%$ of the isolates at a parasite density of $\leq 250$ parasites $/ \mu \mathrm{l}$ were predicted to be undetected by PfHRP2-based RDT. The number of isolates predicted to be non-sensitive to the PfHRP2-based RDT increased significantly between 2009 and 2011 (0\% in 2009, 5\% in 2010 and 16.3\% in 2011; $P=0.0046)$. One hypothesis to explain this increase is the selection of parasites which contain less than 43 repeats (type $2 \times$ type 7 repeats). PfHRP2-based RDTs are now commonly used with high adherence in Senegal [17-19]. Patients with negative PfHRP2-based RDT are not treated for malaria. If the parasites are undetected by the PfHRP2-based RDT, the delay of diagnose before treatment might permit an increase in the time required for development of sexual stages (gametocytes) and in their transmission to mosquitoes during a blood meal compared to parasites treated quickly. The transmission of parasites undetected by the PfHRP2-based RDT might be more important, leading to faster dissemination of these genotypes. The frequency of these genotypes could increase in coming years. This evolution should continue to be monitored in Senegal.

In the Dakar samples with low parasite density, the predicted prevalence was slightly lower than the evaluated prevalence $(7.4 \%$ versus $10.7 \%)$. The predictive regression model developed by Baker cannot explain all of the PfHRP2 non-detection in Senegal. Of the four samples not detected by the PfHRP2-based Palutop $+4^{\circledR}$ but with PfHRP2, only one (183/3) was classified in group C (non-sensitive) according to the Baker's regression model. In addition, of the nine samples expected to be not detected by PfHRP2-based RDT, the sample 183/3 was the only one which was not detected by the PfHRP2based Palutop $+4^{\circledR}$. However, this sample was the only one with parasitaemia $\leq 0.005 \%(\leq 250$ parasites $/ \mu l)$.

It has been reported that, due to PfHRP3 and PfHRP2 structural homology, PfHRP3 can cross-react with HRP2coated antibodies in the RDT. PfHRP3 has also contributed to the detection of $P$. falciparum malaria infections [21]. In this study, the genetic diversity of pfhrp3 (64 different sequences) was less than that of pfhrp2 (120 different sequences). All of the previously described repeat types, other than type 2 , were present ( $99 \%$ to $100 \%$ ). The type 2 repeat has never been described in HRP3 in Africa $[3,4]$. The Senegalese HRP3 sequences had characteristics in common with those of other African isolates [3,4] and were different from Indian isolates in that they did not contain type 1 repeats [6]. The most common repeat types between PfHRP2 and PfHRP3 in Africa are types 1 and 7. These shared repeats, particularly type 7 , which is the only type described both in Africa and India, are likely the basis for the observed cross-reactivity between PfHRP3- and PfHRP2-specific monoclonal antibodies [21,22]. In addition, the type 7 repeat is one of the two selected types in the Baker's regression model to predict non-detection of parasites by PfHRP2 at a density of $\leq 250$ parasites/ $\mu \mathrm{l}$.

\section{Conclusion}

In the present work, $10.7 \%$ of the isolates with a parasitaemia $\leq 0.005 \%$ ( $\leq 250$ parasites $/ \mu \mathrm{l})$ were undetected by the PfHRP2 Palutop $+4^{\circledR}$ RDT $(7.4 \%$ by the predictive Baker'model). In addition, all of the parasites with $p f h r p 2$ deletion $(2.4 \%$ of the total samples) and $2.1 \%$ of the parasites with parasitaemia $>0.005 \%$ and presence of $p f h r p 2$ were not detected by PfHRP2 Palutop $+4^{\circledR}$ RDT. PfHRP2 (and PfHRP3), the most common target of malaria RDTs, is highly polymorphic in Senegal. Future efforts should be made to more accurately determine the prevalence of parasites that are non-sensitive to HRP2-detection-based assays. Better understanding of the structure of PfHRP2 and its diversity at the polymorphism and protein levels will contribute to the evaluation and improvement of malaria RDTs.

\section{Competing interests \\ The authors declare that they have no competing interests.}

\section{Authors' contributions}

NW, KB and AP carried out the diagnostic tests and the molecular genetic studies. BF, MF, CC, BD, KBF, PSM, YD, RB and BW supervised, carried out and coordinated the field collections of patient isolates. NW, SB and BP conceived and coordinated the study. SB and BP analysed the data. NW, AP $\mathrm{SB}$ and BP drafted the manuscript. All the authors read and approved the final manuscript.

\section{Acknowledgements}

The authors thank the patients of Hôpital Principal de Dakar, Ndeye Fatou Diop and Maurice Gomis for technical support and the staff of the clinical units. This work was supported by the Etat Major des Armées Françaises (grant schema directeur paludisme LR 607).

\section{Author details}

${ }^{1}$ Unité de Parasitologie, Département d'Infectiologie de Terrain, Institut de Recherche Biomédicale des Armées, Marseille, France. ${ }^{2}$ Aix Marseille Université, Unité de Recherche sur les Maladies Infectieuses et Tropicales Emergentes, UM 63, CNRS 7278, IRD 198, Inserm 1095, Marseille, France. ${ }^{3}$ Laboratoire d'étude de la chimiosensibilité du paludisme, Fédération des laboratoires, Hôpital Principal de Dakar, Dakar, Sénégal. ${ }^{4}$ Centre National de référence du Paludisme, Marseille, France. ${ }^{5}$ Service de réanimation médicale, Hôpital Principal de Dakar, Dakar, Sénégal. ${ }^{6}$ Service des urgences, Hôpital Principal de Dakar, Dakar, Sénégal. ${ }^{7}$ Service de pathologie infectieuse, Hôpital Principal de Dakar, Dakar, Sénégal. ${ }^{8}$ Département de médecine interne et spécialités médicales de pathologie tropicale, Hôpital Principal de Dakar, Dakar, Sénégal. ${ }^{9}$ Chefferie, Hôpital Principal de Dakar, Dakar, Sénégal. 


\section{References}

1. Murray CK, Gasser RA, Magill AJ, Miller RS: Update on rapid diagnostic testing for malaria. Clin Microbiol Rev 2008, 21:97-110.

2. Lee N, Baker J, Bell D, McCarthy J, Cheng Q: Assessing the genetic diversity of the aldolase genes of Plasmodium falciparum and Plasmodium vivax and its potential effect on performance of aldolasedetecting rapid diagnostic tests. J Clin Microbiol 2006, 44:4547-4549.

3. Mariette N, Barnadas C, Bouchier C, Tichit M, Menard D: Country-wide assessment of the genetic polymorphism in Plasmodium falciparum and Plasmodium vivax antigens detected with rapid diagnostic tests for malaria. Malar J 2008, 7:219.

4. Baker J, Ho MF, Pelecanos A, Gatton M, Chen N, Abdullah S, Albertini A, Ariey F, Barnwell J, Bell D, Cunningham J, Djalle J, Echeverry DF, Gamboa D, Hii J, Kyaw MP, Luchavez J, Membi C, Menard D, Murillo C, Nhem S, Ogutu B, Onyor P, Oyibo W, Wang SQ, McCarthy J, Cheng Q: Global sequence variation in the histidine-rich proteins 2 and 3 of Plasmodium falciparum: implications for the performance of malaria rapid diagnostic tests. Malar J 2010, 9:129.

5. Baker J, McCarthy J, Gatton M, Kyle DE, Belizario V, Luchavez J, Bell D, Cheng Q: Genetic diversity of Plasmodium falciparum histidine-rich protein 2 (PfHRP2) and its effect on the performance of PfHRP2-based rapid diagnostic tests. J Infect Dis 2005, 192:870-877.

6. Kumar N, Singh JPN, Pande V, Mishra N, Srivastava B, Kapor R, Valecha N, Anvikar AR: Genetic variation in histidine rich proteins among Indian Plasmodium falciparum population: possible cause of variable sensitivity malaria of rapid diagnostic tests. Malar J 2012, 11:298.

7. Wurtz N, Briolant S, Lemarié D, Pommier De Santi V, Pascual A, Roodt T, Benoit N, Hupin C, Pradines B: Retard de diagnostic d'un accès palustre à Plasmodium falciparum chez un militaire en Ouganda: négativité des tests de diagnostic rapide associée à un polymorphisme de l'antigène HRP2. Med Sante Trop 2013, accepted (ms120029).

8. Gamboa D, Ho MF, Bendezu J, Torres K, Chiodini PL, Barnwell JW, Incardona S, Perkins M, Bell D, McCarthy J, Cheng Q: A large proportion of $P$. falciparum isolates in the Amazon region of Peru lack pfhrp2 and pfhrp3; implications for malaria rapid diagnostic tests. PLoS One 2010, 5:8091.

9. Maltha J, Gamboa D, Bendezu J, Sanchez L, Cnops L, Gillet P, Jacobs J: Rapid diagnostic tests for malaria diagnosis in the Peruvian Amazon: Impact of pfhrp2 gene deletions and cross-reactions. PLoS One 2012, 7:43094.

10. Koita OA, Doumbo OK, Ouattara A, Tall LK, Konaré A, Diakité M, Diallo M, Sagara I, Masinde GL, Doumbo SN, Dolo A, Tounkara A, Traoré I, Krogstad $D J$ : False-negative rapid diagnosis tests for malaria and deletion of the histidine-rich repeat region of the hrp2 gene. Am J Trop Med Hyg 2012, 86:194-198.

11. Kumar N, Pande V, Bhatt RM, Shah NK, Mishra N, Srivastava B, Valecha N, Anvikar AR: Genetic deletion of HRP2 and HRP3 in Indian P. falciparum population and false negative malaria rapid diagnostic test. Acta Trop 2013, 125:119-121.

12. Houzé S, Hubert V, Le Pessec G, Le Bras J, Clain J: Combined deletions of pfhrp2 and pfhrp3 genes results in Plasmodium falciparum malaria falsenegative rapid diagnostic test. J Clin Microbiol 2011, 49:2694-2696.

13. Fall B, Diawara S, Sow K, Baret E, Diatta B, Ba Fall K, Mbaye PS, Fall F, Diémé $Y$, Rogier R, Wade B, Bercion R, Pradines B: Ex vivo susceptibility of Plasmodium falciparum isolates from Dakar, Senegal, to seven standard anti-malarial drugs. Malar J 2011, 10:310.

14. Gaillard T, Fall B, Tall A, Wurtz N, Diatta B, Lavina M, Ba Fall K, Diene Sarr F, Baret E, Diémé Y, Wade B, Bercion R, Briolant S, Pradines B: Absence of association between ex vivo susceptibility to doxycycline and pftet $Q$ and pfmdt copy numbers in Plasmodium falciparum isolates from Dakar, Senegal. Clin Microbiol Infect 2012, 18:E238-E240.

15. Wurtz N, Fall B, Pascual A, Diawara S, Sow K, Baret E, Diatta B, Fall KB, Mbaye PS, Fall F, Dieme Y, Rogier C, Bercion R, Briolant S, Wade B, Pradines B: Prevalence of molecular markers of Plasmodium falciparum drug resistance in Dakar. Senegal. Malar J 2012, 11:197.

16. Wurtz N, Mint Lekweiry K, Bogreau H, Pradines B, Rogier C, Ould Mohamed Salem Boukhary A, Hafid JE, Ould Ahmedou Salem MS, Trape JF, Basco LK, Briolant S: Vivax in Mauritania includes infection of a Duffy-negative individual. Malar J 2011, 10:336.

17. Ly AB, Tall A, Perry R, Baril L, Badiane A, Faye J, Rogier C, Touré A, Sokhna C, Trape JF, Michel R: Use of HRP-2-based rapid diagnostic test for Plasmodium falciparum malaria: assessing accuracy and cost-effectiveness in the villages of Dielmo and Ndiop, Senegal. Malar J 2010, 9:153.

18. Thiam M, Thior M, Faye B, Ndiop M, Diouf ML, Diouf MB, Diallo I, Ba Fall F, Ndiaye $J$, Albertini $A$, Lee $E$, Jorgensen $P$, Gaye $O$, Bell D: Major reduction in anti-malarial drug consumption in Senegal after nation-wide introduction of malaria rapid diagnostic tests. PLoS One 2011, 3:18419.

19. Thiam S, Thwing J, Diallo I, Fall FB, Diouf MB, Perry R, Diop M, Diouf ML, Cisse MM, Diaw MM, Thior M: Scale-up home-based management of malaria based on rapid diagnostic tests and artemisinin-based combination therapy in a resource-poor country: results in Senegal. Malar J 2012, 11:334.

20. Baker J, Gatton ML, Peters J, Ho MF, McCarthy JS, Cheng Q: Transcription and expression of Plasmodium falciparum histidine-rich proteins in different stages and strains: implications for rapid diagnostic tests. PLOS One 2011, 6:22593.

21. Wellems TE, Howard RJ: Homologous genes encode two distinct histidine-rich proteins in a cloned isolate of Plasmodium falciparum. Proc Natl Acad Sci USA 1986, 83:6065-6069.

22. Sharma YD: Genomic organization, structure and possible function of histidine rich proteins of malaria parasites. Int J Biochem 1988, 20:471-477.

\section{doi:10.1186/1475-2875-12-34}

Cite this article as: Wurtz et al:: Pfhrp2 and pfhrp3 polymorphisms in Plasmodium falciparum isolates from Dakar, Senegal: impact on rapid malaria diagnostic tests. Malaria Journal 2013 12:34.

\section{Submit your next manuscript to BioMed Central and take full advantage of:}

- Convenient online submission

- Thorough peer review

- No space constraints or color figure charges

- Immediate publication on acceptance

- Inclusion in PubMed, CAS, Scopus and Google Scholar

- Research which is freely available for redistribution 